\title{
Analysis of Gene Expression in Mouse Spinal Cord-derived Neural Precursor Cells During Neuronal Differentiation
}

\author{
Joon-lk Ahn, So Young Kim, Moon-Jeong Ko, \\ Hye Joo Chung* and Ho-Sang Jeong*
}

Molecular Pharmacology Division, Pharmacological Research Department, National Institute of Toxicological Research, Seoul 122-704, Korea

\begin{abstract}
The differentiation of neural precursor cells (NPCs) into neurons and astrocytes is a process that is tightly controlled by complicated and ill-defined gene networks. To extend our knowledge to gene networks, we performed a temporal analysis of gene expression during the differentiation (2, 4, and 8 days) of spinal cord-derived NPCs using oligonucleotide microarray technology. Out of 32,996 genes analyzed, 1878 exhibited significant changes in expression level (fold change $>2, p<0.05$ ) at least once throughout the differentiation process. These 1878 genes were classified into 12 groups by k-means clustering, based on their expression patterns. K-means clustering analysis revealed that the genes involved in astrogenesis were categorized into the clusters containing constantly upregulated genes, whereas the genes involved in neurogenesis were grouped to the cluster showing a sudden decrease in gene expression on Day 8. Functional analysis of the differentially expressed genes indicated the enrichment of genes for Pax6NeuroD signaling - TGFb-SMAD and BMP-SMAD - which suggest the implication of these genes in the differentiation of NPCs and, in particular, key roles for Nova1 and TGFBR1 in the neurogenesis/astrogenesis of mouse spinal cord.
\end{abstract}

Keywords: astrogenesis, microarray, neural precursor cells, neurogenesis, spinal cord

\section{Introduction}

Genetic programming of multipotent neural precursor cells (NPCs) of the embryonic spinal cord is a critical prerequisite to the generation of neurons, oligoden-

\footnotetext{
*Corresponding author: E-mail hosa33@kfda.go.kr hjchung@kfda.go.kr

Tel +82-2-380-1804, Fax +82-2-388-6457

Accepted 29 April 2009
}

drocytes, and astrocytes in vivo. The differentiation of NPCs occurs in highly specific manners both in temporal and spatial aspects (Lee et al., 2003) and results in production of neuronal-restricted precursors (NRPs) and glial-restricted precursors (GRPs), which subsequently develop into mature neurons and glial cells, respectively (Kalyani et al., 1998). in vivo, NPCs generate neurons first and then produce mainly astrocytes (Sun et al., 2003; Cheng et al., 2006). This unvarying sequential generation, the so-called gliogenic switch, is reported to be conserved across vertebrates having a central nervous system (CNS), suggesting its fundamental role in neuronal differentiation mechanisms (Deneen et al., 2006). This sequential generation of neurons and astrocytes can be repeated in vitro. It has been reported that when incubated in growth factor-free culture medium, NPCs from early developmental stages differentiate into neurons, whereas those from late embryonic and early postnatal stages differentiate into astrocytes (Qian et al., 2000).

Proliferation of NPCs occurs and stays in the ventricular zone of the neural tube at an early developmental stage. In the presence of intrinsic and environmental signals, some of these precursor cells exit the proliferation cycle and express young neuronal and glial characteristics. The postmitotic cell bodies and differentiating cells are confined within the mantle layer and give rise to a distinct grey appearance. Within this layer, some neurons develop local interconnections, while others elaborate axons to distal targets and form the marginal layer during the process. Glial cells gradually encapsulate the axons producing a marginal layer, which demonstrates a distinctive white appearance in the adult spinal cord. In this way, neuronal and glial cells follow a developmental program that depends on temporal and regional patterning (Poh et al., 2002).

Recent progress in the field has improved our understanding of the genetic control involved in neurogenesis and gliogenesis. For example, the identification of the basic helix-loop-helix (bHLH) transcription factor has allowed us to further understand the molecular regulation of vertebrate neurogenesis and gliogenesis (Bertrand et al., 2002; Kageyama et al., 2005; Lu et al., 2000; Zhou and Anderson, 2002). Despite the identification of intrinsic cellular factors and extracellular cues, the molecular bases of general programs, including sequential generation, are not yet fully understood. In this study, we employed microarray analysis to monitor the tempo- 
ral changes of gene expression during NPC differentiation and to identify the genes playing pivotal roles in the process.

\section{Methods}

\section{Cell culture}

Spinal cords of C57BL/6 mouse embryos at embryonic day 11.5 (E11.5) were dissected in Hank's buffered saline solution (Invitrogen, Carlsbad, CA, USA). Cells from spinal cords were mechanistically dissociated and plated on T-25 or 6-well culture dishes. For neurosphere formation, cells were grown in DMEM/F12 (1:1, Invitrogen) media with $\mathrm{N} 2$ supplement (Invitrogen) at $37^{\circ} \mathrm{C}$ in a $5 \% \mathrm{CO}_{2}$ humidified atmosphere in the presence of $20 \mathrm{ng} / \mathrm{ml}$ epidermal growth factor (EGF; Invitrogen) and basic fibroblast growth factor (bFGF, Invitrogen) for 4 days. EGF and bFGF were added to the culture medium every 2 days. Subsequently, the culture medium containing floating neurospheres was collected in sterile $15 \mathrm{ml}$ tubes and centrifuged at 110 relative centrifugal forces for $5 \mathrm{~min}$. The neurosphere pellets were dissociated with trypsin, and single cells were seeded at a density of approximately $4 \times 10^{4} \mathrm{cells} / \mathrm{cm}^{2}$ in 6-well culture dishes or $12-\mathrm{mm}$ glass cover slips coated with poly-L-ornithine $(0.1 \mathrm{mg} / \mathrm{ml}$, Sigma-Aldrich, St. Louis, MO, USA) and fibronectin (1 ug/ml, SigmaAldrich). Cells in the culture dishes were allowed to attach during growth in the same medium for 2 days. Differentiation was induced by withdrawal of bFGF and EGF. These spinal cord precursor cells were incubated under the differentiation conditions for 8 days.

\section{Immunocytochemistry}

Spinal cord precursor and differentiated cells were fixed in $4 \%$ paraformaldehyde-containing phosphate-buffered saline (PBS) at room temperature for $10 \mathrm{~min}$. After rinsing several times with PBS, the cells were incubated for 10 min in PBS containing $0.4 \%$ Triton $\mathrm{X}-100$ and rerinsed with PBS containing $0.1 \%$ Tween-20 (TPBS). Then, the cells were incubated for $2 \mathrm{~h}$ with $10 \%$ horse serum or $10 \%$ goat serum in TPBS. To reduce nonspecific antibody binding, the cells were incubated overnight at $4^{\circ} \mathrm{C}$ with primary antibodies depending on conditions; mouse monoclonal anti-nestin (1:100, Chemicon, Temecula, CA, USA), mouse monoclonal Tuj1 (1:100, Covance, East Lansing, MI, USA), low-affinity neurotrophin receptor (p75, 1:100, Chemicon), and GFAP (1:100, DAKO, Glostrup, Denmark). After rinsing with TPBS 3 times, the cells were incubated with fluorescein isothiocyanate- or Texas red-conjugated secondary anti- body (1:100, Vector Laboratories, Burlingame, CA, USA) for $2 \mathrm{~h}$ at room temperature. Cell nuclei were counterstained with 4', 6-diamidino-2-phenylindole (DAPI; Vector Laboratories) for $10 \mathrm{~min}$ at room temperature. Fluorescent images were captured with a Leica DM IRB microscope (Leica Microsystems, Wetzlar, Germany)

\section{RNA isolation and DNA microarray}

Total RNA was extracted using Trizol reagent (Invitrogen) in accordance with the manufacturer's manual. Quantification and determination of purity (260/280 ratio) of RNA were performed using an ND-1000 UV/VIS spectrophotometer (NanoDrop Technologies, Wilmington, DE, USA). A commercial mouse genome survey array was used for differential gene expression profiling (Applied Biosystems, Foster City, CA, USA). The array consists of 60-mer oligonucleotide probes, representing a set of 32,996 individual mouse genes and more than 1000 control probes. Microarray experiments were performed following the manufacturer's instructions. Briefly, digoxigenin (DIG)-UTP-labeled cRNA was generated by amplification of $5 \mu \mathrm{g}$ of total RNA using a chemiluminescent reverse transcription in vitro transcription labeling kit (Applied Biosystems). Each microarray was prehybridized in hybridization buffer with blocking reagent at $55^{\circ} \mathrm{C}$ for $1 \mathrm{~h}$. Hybridization of arrays was then carried out using DIG-labeled cRNA $(10 \mu \mathrm{g})$, fragmented into sizes of $100-400 \mathrm{bp}$, at $55^{\circ} \mathrm{C}$ for $16 \mathrm{~h}$. The arrays were washed with hybridization wash buffer, followed by a subsequent wash with chemiluminescence rinse buffer. Chemiluminescent signals were generated by incubating the arrays with anti-DIG alkaline phosphatase and chemiluminescence substrate. Images were collected for each microarray using the Model 1700 Chemiluminescent Microarray Analyzer (Applied Biosystems). Microarray images were autogrided, and the chemiluminescent signals were quantified, corrected for background, and spatially normalized.

\section{Analysis of microarray expression data}

Signal intensities were imported into GenPlex software (Istech, Korea) and normalized to reduce interarray variation in hybridization intensity after filtering out probe sets with unreliable intensity values (flag value $<100$ and $\mathrm{S} / \mathrm{N}<2$ ). Expression changes were presented as fold-changes in comparison with intensities from the control group. The expression intensities were then $\log _{2}$-transformed. We took the average value from the gene expression ratio obtained in 3 biological replicates. DEGs were selected on the basis of ratios (fold change $>2)$ and Welch's t-test $(p<0.05)$. For further analysis, 
Table 1. RT-PCR primer sequences

\begin{tabular}{lll}
\hline Gene name & \multicolumn{1}{c}{ Forward } & \multicolumn{1}{c}{ Reverse } \\
\hline Nkx2.2 & GGAGGACTCGATCCTTACCAC & AGTCAATGGAATCTGCCA \\
Pax6 & GGACCACTCAACAGGACTCA & TTTCCTCTCTCGATCACATGC \\
Neurod6 & GAAATACCTCCCCTGAGTGTG & CTGCGCAGATGTAAGTCGTAAG \\
Ntrk2 & TTCCGCTAGGATTTGGTTAC & GAGCAGCCAGACGTGCAGATG \\
BMP4 & TGTGAGGAGTTTCATCACG & TTATTCTTCTTCCTGGACCG \\
Ncam2 & GGCATCAGAAACCTGGAAAA & CCTCCATCATCTTGCTTGGT \\
Nova1 & CTAGCAGCCAGTGCAGTTTCTA & GGGCAAACTCAGAGGTTAACAG \\
TGFBR1 & TGAGTCACTGGTTTTATGAGG & ACTTAATCTGCAGCGAGACC \\
$\beta$-actin & CCATCATGAAGTGTGACGTTG & GTGCTAGGAGCCAGAGCAGTA \\
\hline
\end{tabular}

A

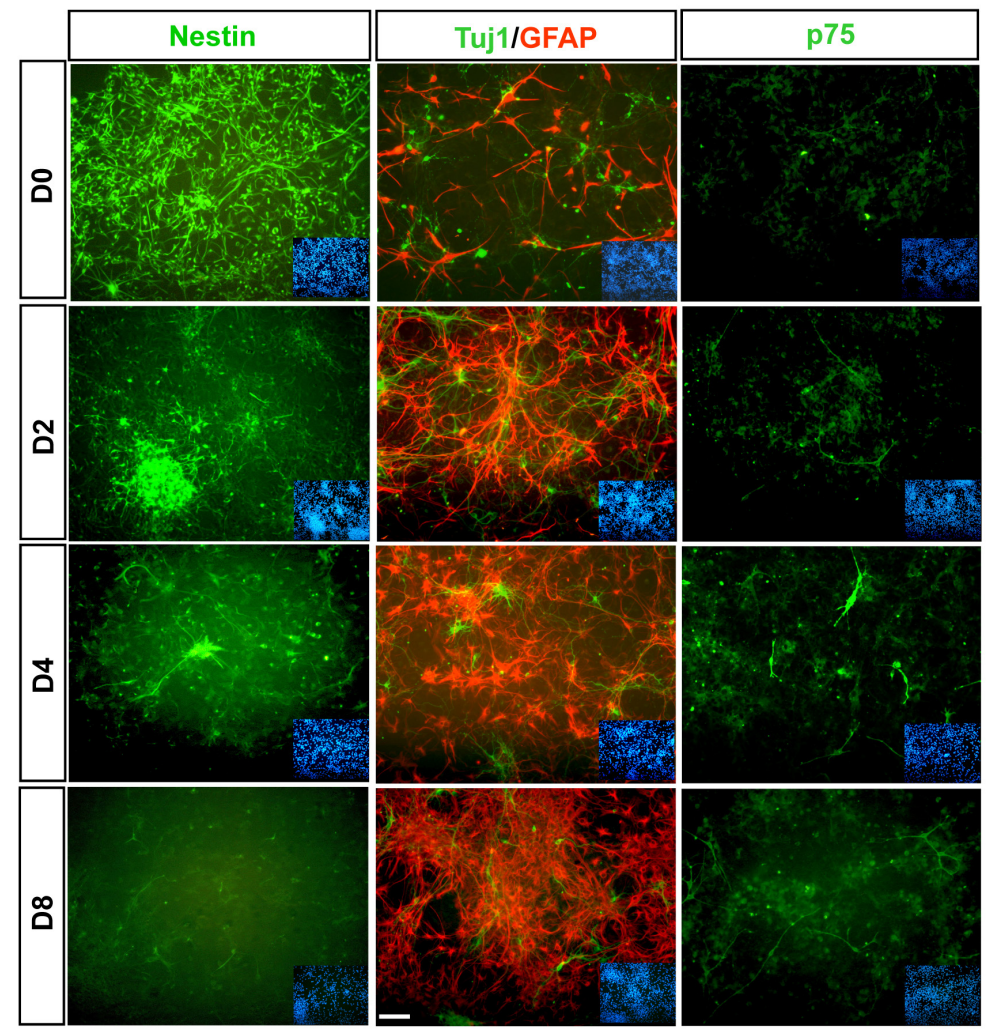

B

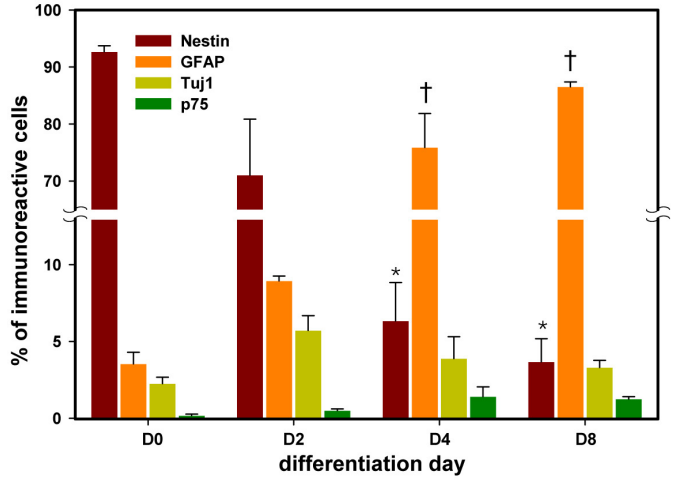

Fig. 1. Differentiation of spinal cord-derived NPCs. (A) Immunocytochemistry of NPCs, neurons, astrocytes, and motoneurons. (B) DAPI nuclear staining results. Most cells at Day 0 were immunostained in green with the intermediate filament nestin, an NPC marker. Differentiation of NPCs was induced by withdrawal of bFGF and EGF. After 2, 4, and 8 days of bFGF and EGF removal, differentiated cells were immunostained with Tuj1 (green), the neuron marker GFAP (red), and the astrocyte and motoneuron marker p75 (green). The results shown in the graph are percentages (mean \pm SEM; $n=3$ ) of nestin-, Tuj1-, GFAP-, and p75-immunoreactive cells relative to total cell numbers. Scale bar, $50 \mu \mathrm{m}$. ${ }^{*} \mathrm{p}<0.05$ and ${ }^{\dagger} \mathrm{p}<0.05$, significantly different from differentiation at Day 0 . One-way analysis of variance (ANOVA) was applied.

DEGs were divided into gene clusters according to expression levels at each time point (Chung et al., 2007; Ko et al., 2008). K-means clustering was applied to genes using the Euclidean distance as a similarity measurement, as implemented in the Genesis software program (http://genome.tugraz.at). Additionally, DEGs were categorized according to their biological processes using the PANTHER (Protein ANalysis THrough Evolutionary Relationships) protein classification system (Applied Biosystems, https://panther.appliedbiosystems.com). 


\section{Protein interaction network analysis}

To further define the molecular mechanism involved in the differentiation of spinal cord precursor cells, Cytoscape ver. 2.5 software was used for protein interaction analysis to identify potential regulatory genes and pathways. The molecular interaction data were downloaded from the BIND and BIOGRID websites. Each dataset was loaded separately to the software, and 2 distinct protein-protein interaction networks were generated. For each network, the DEGs were mapped, and the proteins that interacted directly with the DEGs were selected to generate groups of subnetworks. The subnetworks were merged into a single subnetwork using the "union" function in Cytoscape, and the nodes interacting with more than 9 proteins were selected.

\section{Verification of microarray}

For semiquantitative RT-PCR, beta-actin was used as an internal control. For each sample, the same total RNA that was used for microarray hybridization was reverse-transcribed using $1 \mu \mathrm{M}$ oligo (dT) primer with
Superscript II reverse transcriptase (Invitrogen). The thermal profiles consisted of $95^{\circ} \mathrm{C}$ for $5 \mathrm{~min}$ for initial denaturing, followed by $25 \sim 30$ cycles of $95^{\circ} \mathrm{C}$ for $30 \mathrm{~s}, 55 \sim$ $60^{\circ} \mathrm{C}$ for $30 \mathrm{~s}$, and $72^{\circ} \mathrm{C}$ for $30 \mathrm{~s}$ to $1 \mathrm{~min}$. All RT-PCR reactions were repeated at least 3 times. The primers used in this study are listed in Table 1.

\section{Results and Discussion}

\section{Verification of spinal cord precursor cells into} neuronal cells by immunocytochemistry

Prior to gene expression study of spinal cord precursor cells, their differentiation process was monitored by immunocytochemical detection using the most representative neural markers, such as nestin, Tuj1, and GFAP. The differentiation was initiated by removing basic fibroblast growth factor (bFGF) from the medium on Day 0 , and the process was monitored on Days 2, 4, and 8 after the initiation (Fig. 1).

On Day 0 , most cells in the culture $(92.6 \pm 1.1 \%)$ were nestin-positive, a common characteristic for NPCs, whereas only a small portion (less than $2.5 \%$ and $3.5 \%$ )

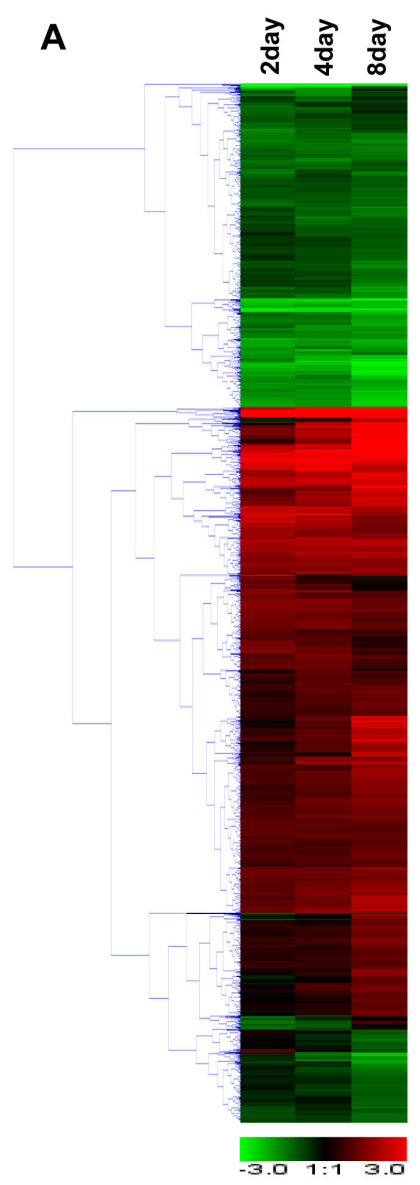

\section{B}

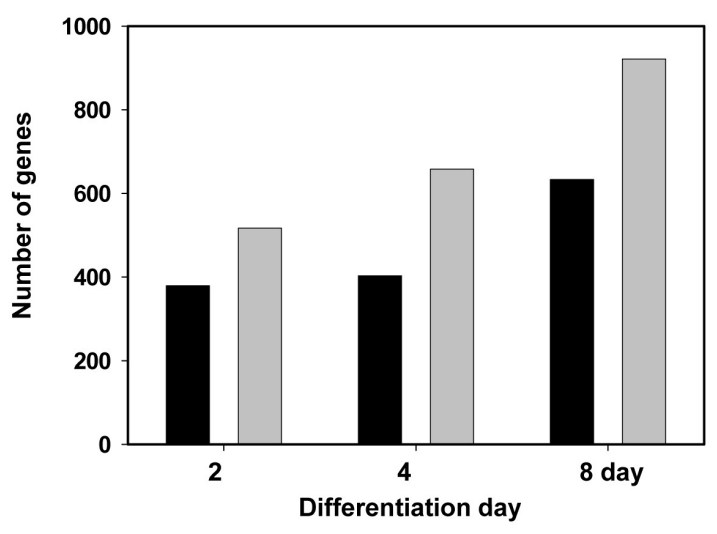

Fig. 2. (A) Hierarchical clustering of 1878 genes. These genes were clustered hierarchically on the basis of the similarity of their expression profiles. The intensity of red and green is proportional to the relative gene induction (red) or repression (green). (B) Number of differentially expressed genes on each differentiation day. Black bar represents downregulation and grey bar represents upregulation. 
of the total cells were found to be tubulin $\beta$ III (Tuj1)positive and glial fibrillary acidic protein (GFAP)-positive, specific characteristics for neurons and astrocytes, respectively. As the differentiation progressed, the proportion of nestin-positive cells in the culture decreased subsequently to $71.0 \pm 9.8 \%, 6.3 \pm 2.5 \%$, and $3.7 \pm 1.5 \%$ on Days 2, 4, and 8, respectively. In the meantime, the proportion of Tuj1-positive cells reached $5.7 \pm 1.7 \%$ of total cells on Day 2 and decreased gradually thereafter $(3.9 \% \pm 1.4 \%$ on Day 4 and $3.3 \pm 0.4 \%$ on Day 8$)$. It was noticed that a small portion of the Tuj1-positive cells were also immunoreactive to anti-p75 antibody on Days 2, 4, and 8, which is a characteristic of motoneurons in the developing neural tube (Camu and Henderson, 1992). The number of GFAP-positive cells increased along the differentiation process, representing $8.9 \pm 0.3 \%$ on Day 2, $75.9 \pm 6.0 \%$ on Day 4 , and $86.5 \pm$ $0.8 \%$ of total cells on Day 8 . This observation coincides well with a previous report (Kalyani et al., 1997), in which GFAP-positive cells constituted the largest portion $(73 \pm 6.0 \%)$ of the total population of differentiated neuroepithelial cells after 5 days of differentiation.

Based on the immunocytochemical observation pre- sented in this study, it was confirmed that spinal cord precursor cells sequentially generate neurons and astrocytes along the process, the so-called gliogenic switch, as described in a previous report (Qian et al., 2000).

\section{Identification of differentially expressed genes}

To examine the temporal changes in gene expression along the differentiation, the expression level of the whole genome was profiled using a microarray. Total RNA from cells on Day 0, Day 2, Day 4, and Day 8 of differentiation was subjected to microarray analysis. Gene expression levels in NPCs (Day 0) were compared with those in differentiated cells (Days 2, 4, and 8). Out of the 32,996 genes represented on the chip, 1878 were differentially expressed more than 2-fold at least at one point during the experiment (Fig. 2A and Supplemental data). On Day 2, 517 genes were upregulated and 379 genes were downregulated. On Day 4, 658 genes were upregulated and 403 genes were downregulated. On Day 8, when most spinal cord precursor cells were differentiated into astrocytes, 1554 genes showed modu-

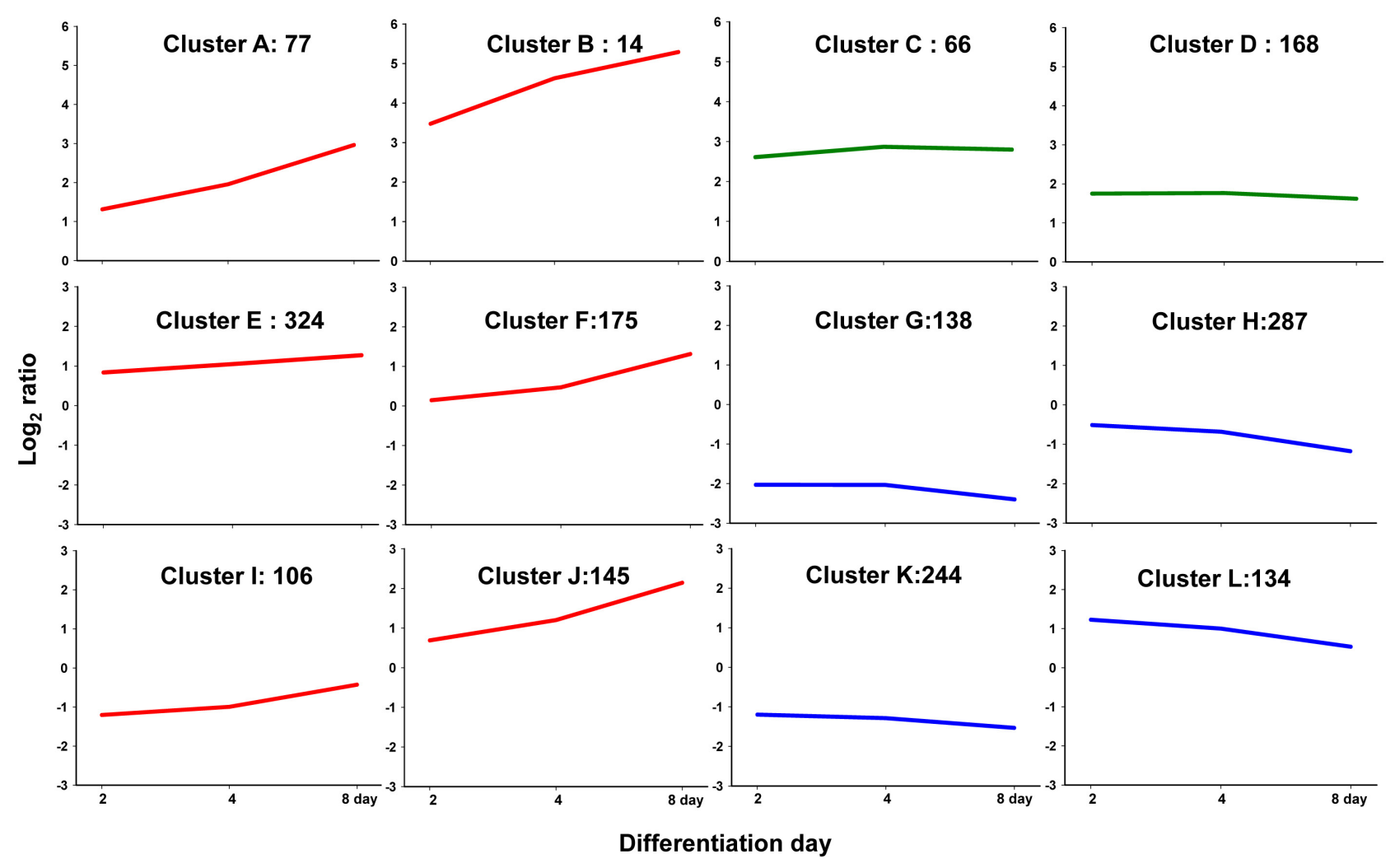

Fig. 3. K-means clustering of genes exhibiting altered expression levels during NPC differentiation. The 1878 genes were classified into 12 clusters based on similar expression over the 3 time points. The number at the center of each cluster inset represents the number of genes in that particular cluster. Lines indicate the mean expression value. 
lated expression levels (Fig. 2B).

\section{K-means clustering analysis of differentially ex- pressed genes}

To characterize the patterns of gene expression along the time course of differentiation, k-means clustering was performed on the 1878 differentially expressed genes. The genes were classified into 12 clusters (Fig. 3). Six clusters showed a gradual increase by Day 8 .
The expression of genes in clusters $A$ and $B$ was upregulated more than 2-fold at all time points. In particular, Cluster B comprised highly upregulated genes (>eight folds) and included the astrocyte-specific marker GFAP. Clusters $C$ and $D$ consisted of genes that were constantly upregulated more than 2-fold at all time points. Four clusters contained genes with decreasing expression as the differentiation progressed. Clusters $G$ and $\mathrm{K}$ comprised genes whose expression decreased more than 2 -fold at all time points.

Table 2. Abundance of differentially expressed genes categorized by their biological function in each cluster

\begin{tabular}{|c|c|c|c|c|c|}
\hline PANTHER classification category & Cluster & $\begin{array}{c}\text { Number of DEGs } \\
\text { in array }\end{array}$ & $\begin{array}{c}\text { Number of } \\
\text { changed genes in } \\
\text { each cluster }\end{array}$ & Expected value & $p$ value \\
\hline Neuromuscular synaptic transmission & $A$ & 4 & 2 & 0.17 & 0.012 \\
\hline Antioxidation & $A$ & 5 & 2 & 0.21 & 0.018 \\
\hline Transport & $\mathrm{B}$ & 91 & 3 & 0.74 & 0.034 \\
\hline Apoptosis & $\mathrm{B}$ & 39 & 2 & 0.32 & 0.039 \\
\hline Neuronal activities & $\mathrm{C}$ & 61 & 6 & 2.13 & 0.019 \\
\hline Homeostasis & $\mathrm{C}$ & 18 & 3 & 0.63 & 0.025 \\
\hline Intracellular signaling & $\mathrm{C}$ & 21 & 3 & 0.73 & 0.037 \\
\hline Cell structure & $\mathrm{C}$ & 54 & 5 & 1.88 & 0.039 \\
\hline Neuronal activities & $\mathrm{D}$ & 61 & 12 & 4.99 & $4.5 \mathrm{e}-3$ \\
\hline Cell adhesion-mediated signaling & $\mathrm{D}$ & 34 & 7 & 2.78 & 0.022 \\
\hline Cell adhesion & $\mathrm{D}$ & 65 & 10 & 5.32 & 0.041 \\
\hline Synaptic transmission & $\mathrm{D}$ & 31 & 6 & 2.54 & 0.043 \\
\hline Developmental processes & $\mathrm{D}$ & 207 & 24 & 16.95 & 0.043 \\
\hline Coenzyme metabolism & $\mathrm{E}$ & 12 & 8 & 2.07 & $1.2 \mathrm{e}-3$ \\
\hline Lipid metabolism & $\mathrm{E}$ & 75 & 25 & 12.94 & $1.4 \mathrm{e}-3$ \\
\hline Lipid and fatty acid transport & $\mathrm{E}$ & 16 & 9 & 2.76 & $2.0 \mathrm{e}-3$ \\
\hline Immunity and defense & $\mathrm{F}$ & 97 & 22 & 9.46 & $2.1 \mathrm{e}-4$ \\
\hline Carbohydrate metabolism & $\mathrm{F}$ & 53 & 15 & 5.17 & $2.4 \mathrm{e}-4$ \\
\hline Detoxification & $\mathrm{F}$ & 7 & 5 & 0.68 & $6.7 \mathrm{e}-4$ \\
\hline Sulfur metabolism & $\mathrm{F}$ & 7 & 4 & 0.68 & $5.1 \mathrm{e}-3$ \\
\hline Phospholipid metabolism & $\mathrm{F}$ & 18 & 5 & 1.76 & 0.032 \\
\hline Cell cycle & $\mathrm{G}$ & 110 & 36 & 8.69 & $1.7 \mathrm{e}-13$ \\
\hline DNA metabolism & $\mathrm{G}$ & 47 & 12 & 3.71 & $3.7 \mathrm{e}-4$ \\
\hline Extracellular matrix-mediated signaling & $\mathrm{G}$ & 9 & 4 & 0.71 & $5.9 e-3$ \\
\hline Protein phosphorylation & G & 63 & 10 & 4.98 & 0.028 \\
\hline Nucleic acid metabolism & $\mathrm{H}$ & 266 & 75 & 41.55 & $1.4 \mathrm{e}-7$ \\
\hline Oncogene & 1 & 9 & 3 & 0.5 & 0.014 \\
\hline Immunity and defense & I & 97 & 11 & 5.41 & 0.019 \\
\hline Immunity and defense & $\mathrm{J}$ & 97 & 16 & 7.6 & $4.0 \mathrm{e}-3$ \\
\hline Proteolysis & J & 64 & 11 & 5.02 & 0.012 \\
\hline Nucleic acid metabolism & $\mathrm{K}$ & 266 & 65 & 35.99 & $9.2 \mathrm{e}-7$ \\
\hline DNA metabolism & $\mathrm{K}$ & 47 & 20 & 6.36 & $7.9 \mathrm{e}-6$ \\
\hline Cell cycle & $\mathrm{K}$ & 110 & 32 & 14.88 & $4.0 e-5$ \\
\hline Nuclear transport & $\mathrm{K}$ & 13 & 6 & 1.76 & $9.0 \mathrm{e}-3$ \\
\hline Cell structure and motility & $\mathrm{L}$ & 98 & 19 & 5.75 & $3.9 \mathrm{e}-6$ \\
\hline Cell adhesion & L & 65 & 13 & 3.81 & $1.1 \mathrm{e}-4$ \\
\hline Neurogenesis & L & 77 & 12 & 4.52 & $1.8 \mathrm{e}-3$ \\
\hline Signal transduction & $\mathrm{L}$ & 270 & 24 & 15.84 & 0.022 \\
\hline
\end{tabular}

Representative groups with $p$ value lower than 0.05 in each cluster are listed. Expected value is the number of genes that would be expected for a particular PANTHER category, based on 1878 differentially expressed genes (reference list). $p$ value was determined by the binomial statistic. 


\section{Functional enrichment analysis of K-means clus- tered genes}

To identify genes involved in the differentiation of spinal cord precursor cells, it was of interest to apply an ontological study to the clustered genes. For this purpose, functional enrichment analyses were performed on the differentially expressed genes grouped in each cluster. Table 2 summarizes the list of biological functions that were overrepresented among the differentially expressed genes in each cluster. Based on the enrichment analysis, only clusters $A, C, D$, and $L$ showed significant enrichment $(p<0.05)$ of biological functions related to neuronal development, such as development/developmental processes, neuronal activities, neurogenesis, and synaptic transmission. The list of genes that contributed to enrichment of biological function in clusters $A, C, D$, and $L$ is summarized in Table 3 .

In cluster $\mathrm{C}$, in which most genes were constantly upregulated more than 2-fold at all time points, we noticed the presence of basic helix-loop-helix domain-containing class B5 (Bhlhb5). In a previous report, the Bhlhb5 gene was identified as a member of a new subclass of the bHLH family (Bramblett et al., 2002; McLellan et al., 2002). The proteins in this class, as well as members of the HES and ID families, are considered negative regulators of the function and/or transcription of other bHLH proteins (e.g., Mash1, Math, and Neurogenin2) that are necessary and sufficient to promote neuronal differentiation (Kageyama et al., 2005). It has been also reported that the inhibitory HLH factors Id1 and Hes1 stimulate astrogenesis in vitro (Sugimori et al., 2007) and that mouse Bhlhb5 represses a human Pax6 promoter (Xu et al., 2002). Bhlhb5, constantly upregulated along the differentiation, suggests a possible implication of this protein during gliogenic differentiation as a transcriptional regulator.

Cluster D, which consisted of 168 constantly upregulated genes, showed significant enrichment of genes related to biological functions, such as neuronal activities, synaptic transmission, and developmental processes (Table 2). Among the genes that belong to enriched biological functions in cluster $\mathrm{D}, \mathrm{Nkx2.2}$ and BMP/SMAD have been shown to promote gliogenesis (Lee et al., 2003; Nakashima et al., 2001; Fukuda et al., 2007). In addition, the expression of truncated tyrosine kinase $B$ (trkB-t) has been reported to play a pivotal role in the developmental transition from neurogenesis to gliogenesis (Cheng et al., 2006; Climent et al., 2000). These reports strongly suggest a possible association of the

Table 3. List of neuromuscular synaptic transmission-, neuronal activity-, synaptic transmission-, developmental process-, and neurogenesis-related genes in each cluster

\begin{tabular}{|c|c|c|c|c|}
\hline PANTHER classification category & Cluster & Probe id & Gene name & Symbol \\
\hline \multirow[t]{2}{*}{ Neuromuscular synaptic transmission } & A & 377521 & Syntrophin, acidic 1 & Snta1 \\
\hline & A & 629403 & Dystrobrevin alpha & Dtna \\
\hline \multirow[t]{6}{*}{ Neuronal activities } & $\mathrm{C}$ & 338995 & Solute carrier family 1 , member 1 & Slc1a1 \\
\hline & $\mathrm{C}$ & 749790 & Syntrophin, gamma 2 & Sntg2 \\
\hline & C & 494196 & Doublecortin and calcium/calmodulin-dependent protein kinase-like 1 & Dcamkl1 \\
\hline & C & 336928 & Basic helix-loop-helix domain containing, class B5 & Bhlhb5 \\
\hline & C & 718185 & Cerebellin 2 precursor protein & Cbln2 \\
\hline & C & 533127 & SH3-domain GRB2-like 3 & Sh3gl3 \\
\hline \multirow[t]{12}{*}{ Neuronal activities } & $\mathrm{D}$ & 776947 & Phosphatase and actin regulator 1 & Phactr1 \\
\hline & $\mathrm{D}$ & 452059 & Gamma-aminobutyric acid (GABA-B) receptor, 1 & Gabbr1 \\
\hline & $\mathrm{D}$ & 925263 & Synaptic vesicle glycoprotein 2 a & Sv2a \\
\hline & $\mathrm{D}$ & 854132 & Synaptoporin & Synpr \\
\hline & $\mathrm{D}$ & 889212 & RAB3A interacting protein (rabin3)-like 1 & Rab3il1 \\
\hline & $\mathrm{D}$ & 833456 & Neural cell adhesion molecule 2 & Ncam2 \\
\hline & $\mathrm{D}$ & 786106 & Sortilin-related VPS10 domain containing receptor 3 & Sorcs3 \\
\hline & $\mathrm{D}$ & 377670 & Fasciculation and elongation protein zeta 1 & Fez1 \\
\hline & $\mathrm{D}$ & 803105 & Neuroligin 1 & Nlgn1 \\
\hline & $\mathrm{D}$ & 608088 & Syntrophin, gamma 1 & Sntg1 \\
\hline & $\mathrm{D}$ & 931645 & Plasma membrane proteolipid & Pllp \\
\hline & $\mathrm{D}$ & 852817 & Neurexin III & Nrxn3 \\
\hline \multirow[t]{5}{*}{ Synaptic transmission } & D & 452059 & Gamma-aminobutyric acid (GABA-B) receptor, 1 & Gabbr1 \\
\hline & $\mathrm{D}$ & 925263 & Synaptic vesicle glycoprotein 2 a & Sv2a \\
\hline & $\mathrm{D}$ & 854132 & Synaptoporin & Synpr \\
\hline & $\mathrm{D}$ & 608088 & Syntrophin, gamma 1 & Sntg1 \\
\hline & $\mathrm{D}$ & 889212 & RAB3A interacting protein (rabin3)-like 1 & Rab3il1 \\
\hline
\end{tabular}


92 Genomics \& Informatics Vol. 7(2) 85-96, June 2009

Table 3. Continued

\begin{tabular}{|c|c|c|c|c|}
\hline PANTHER classification category & Cluster & Probe id & Gene name & Symbol \\
\hline \multirow[t]{25}{*}{ Developmental processes } & D & 852817 & Neurexin III & Nrxn3 \\
\hline & D & 930146 & Four and a half LIM domains 1 & Fhl1 \\
\hline & D & 370481 & Down syndrome cell adhesion molecule-like 1 & Dscaml1 \\
\hline & D & 530589 & Bone morphogenetic protein 4 & Bmp4 \\
\hline & D & 785886 & Cytoplasmic polyadenylation element binding protein 2 & Cpeb2 \\
\hline & D & 803105 & Neuroligin 1 & Nlgn1 \\
\hline & D & 827822 & Very low density lipoprotein receptor & VIdlr \\
\hline & D & 744253 & Tensin 3 & Tns3 \\
\hline & D & 506452 & Prickle like 1 (Drosophila) & Prickle1 \\
\hline & D & 350633 & Amyloid beta (A4) precursor-like protein 1 & Aplp1 \\
\hline & D & 753186 & Tripartite motif protein 2 & Trim2 \\
\hline & $\mathrm{D}$ & 827245 & Thrombospondin, type I, domain containing 7A & Thsd7a \\
\hline & D & 550580 & Cytoplasmic FMR1 interacting protein 2 & Cyfip2 \\
\hline & D & 870866 & Leucine rich repeat neuronal $6 \mathrm{C}$ & Lrrn6c \\
\hline & D & 352965 & t-complex 11 (mouse) like 2 & Tcp1112 \\
\hline & $\mathrm{D}$ & 437292 & MAD homolog 7 (Drosophila) & Smad7 \\
\hline & D & 930146 & Four and a half LIM domains 1 & Fhl1 \\
\hline & D & 641040 & Protocadherin beta 14 & Pcdhb14 \\
\hline & $\mathrm{D}$ & 729136 & Protocadherin beta 15 & Pcdhb15 \\
\hline & D & 651940 & Protocadherin beta 20 & Pcdhb20 \\
\hline & $\mathrm{D}$ & 833456 & Neural cell adhesion molecule 2 & Ncam2 \\
\hline & D & 626399 & $\begin{array}{l}\text { Sema domain, transmembrane domain }(\mathrm{TM}) \text {, } \\
\text { and cytoplasmic domain, (semaphorin) } 6 \mathrm{~A}\end{array}$ & Sema6a \\
\hline & D & 342710 & Neurotrophic tyrosine kinase, receptor, type 2 & Ntrk2 \\
\hline & D & 371284 & NK2 transcription factor related, locus 2 (Drosophila) & Nkx2-2 \\
\hline & D & 586957 & DIX domain containing 1 & Dixdc1 \\
\hline \multirow[t]{12}{*}{ Neurogenesis } & $\mathrm{L}$ & 586936 & Reticulon 4 & Rtn4 \\
\hline & $\mathrm{L}$ & 612748 & Neurogenic differentiation 6 & Neurod6 \\
\hline & L & 456676 & Sema domain (semaphorin) $5 \mathrm{~A}$ & Sema5a \\
\hline & L & 681156 & Roundabout homolog 2 (Drosophila) & Robo2 \\
\hline & L & 621590 & Eph receptor B1 & Ephb1 \\
\hline & $\mathrm{L}$ & 760825 & Cadherin EGF LAG seven-pass G-type receptor 1 & Celsr1 \\
\hline & L & 508826 & Contactin 5 & Cntn5 \\
\hline & L & 596198 & Sidekick homolog 2 (chicken) & Sdk2 \\
\hline & L & 331509 & Kelch-like 25 (Drosophila) & $\mathrm{KIhl} 25$ \\
\hline & $\mathrm{L}$ & 923718 & Cadherin 11 & Codh11 \\
\hline & L & 646184 & Brevican & Bcan \\
\hline & L & 377059 & Sema domain (semaphorin) $5 \mathrm{~A}$ & Sema5a \\
\hline
\end{tabular}

upregulation of these genes (Nkx2.2, BMP4, Smad7, Ntrk-2) with gliogenic differentiation of spinal cord precursor cells.

Neurogenesis-related genes were found to be highly enriched in cluster $L$, which showed declining upregulation patterns along the differentiation time. In particular, the Neurod6 gene was upregulated on Days 2 and 4 but decreased on Day 8 in our experiments. Neurod6, also called Nex, Atoh2, and Math2, is a member of the NeuroD family of bHLH transcription factors (Guo et al., 2002) and is a critical effector of the nerve growth factor (NGF) pathway (Schwab et al., 2000). It is also involved in neuronal differentiation in PC12 cells and may be one of the executors of neurogenic function of Pax6 (Holm et al., 2007). It has been reported that Pax6 may be in- volved in the neuronal commitment of the spinal cord and induces the expression of Ngn2, a proneuronal gene, in the mouse spinal cord (Scardigli et al., 2001). Robo2 and Sema5a, known as axon guidance molecules (Yue et al., 2006; Kantor et al., 2004), are reported to be regulated by Pax6 function (Jones et al., 2002; Jiménez et al., 2002). Based on these early reports, Pax6-NeuroD6 and its downstream signaling seem to have important roles in neurogenic differentiation of the spinal cord.

The immunocytochemistry study in the earlier section revealed that there was significant differentiation of the astrocyte on Day 4. But the expression analysis in cluster $D$ showed genes involved in astrocyte differentiation, such as Nkx2.2, BMP, Smad7, and NtrK-2, on Day 2 as 
well as on Day 4. This observation implies the existence of a repression mechanism of astrocyte differentiation in the early stage between Day 2 and Day 4. It was also in agreement with a previous report in which Ngn1 was suggested to inhibit astrocyte differentiation and promote neurogenesis in midgestational NPCs (Sun et al., 2001). Any significant change in expression of $\mathrm{Ngn} 1$ was not observed in the experiment, but Neurod6, one of the bHLH factors, showed significantly increased expression in the early stage of differentiation. To confirm whether neurod6 is able to repress astrocyte differentiation, further investigation might be necessary.

To verify the expression of genes, including those mentioned in this section, 5 selected genes (Lmo2, Nkx2.2, BMP4, Ncam2, NeuroD6) were subjected to semiquantitative RT-PCR analysis. The expression pattern of each gene was consistent with the results of the microarray (Fig. 4).

\section{Computational biomolecule interaction network analysis}

For a better understanding of the underlying mechanism of spinal cord precursor cell differentiation, a biological interaction network was constructed using the Biomolecular Interaction Network Database (BIND; http://bond. unleashedinformatics.com/) and the Biological General Repository for Interaction Datasets (BIOGRID; http:// www.thebiogrid.org/) and visualized with Cytoscape v2.5 open source software (http://www.cytoscape.org). The network obtained from BIND was composed of 2584 edges (interactions) and 2054 nodes (genes), and the network from BIOGRID was composed of 658 interactions and 424 genes. A single subnetwork was constructed initially by mapping both differentially ex- pressed genes (DEGs) and their first neighbors for each time point. Up- and downregulated genes were colored

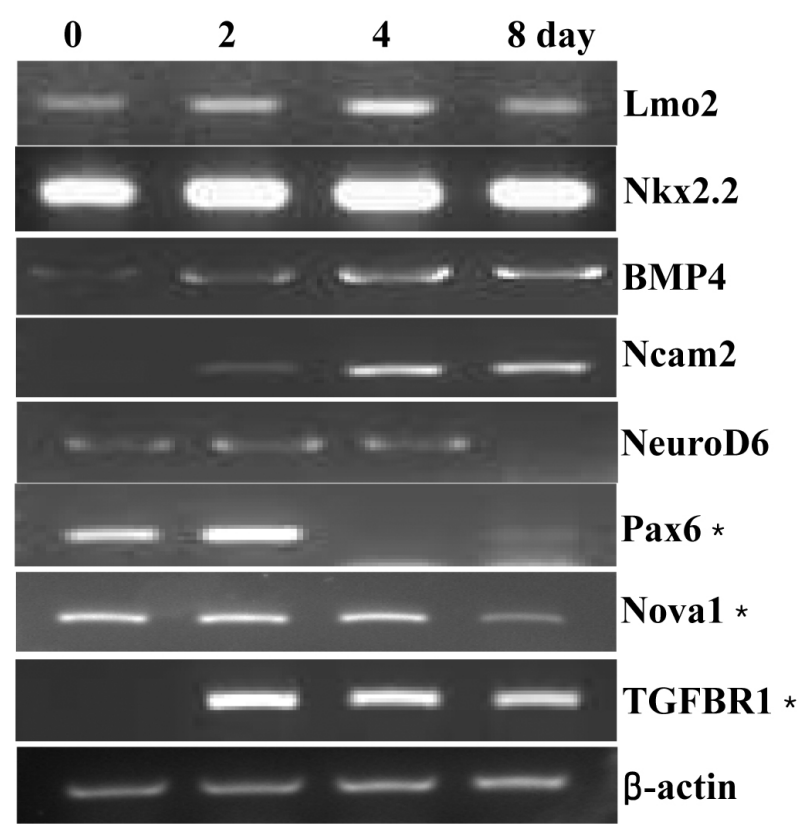

Fig. 4. Verification of microarray data by RT-PCR. Semiquantitative RT-PCR was performed to verify the relative mRNA levels of genes measured by microarray hybridization. Five genes analyzed by microarray and 3 other genes of interest $\left({ }^{*}\right)$ were assayed by RT-PCR. Lmo2, LIM domain only 2; Nkx2.2, NK2 transcription factor related, locus 2; BMP4, bone morphogenetic protein 4; Ncam2, neural cell adhesion molecule 2; NeudoD6, neurogenic differentiation 6; Pax6, paired box gene 6; Nova1, neuro-oncological ventral antigen 1; TGFBR1, transforming growth factor beta receptor I.
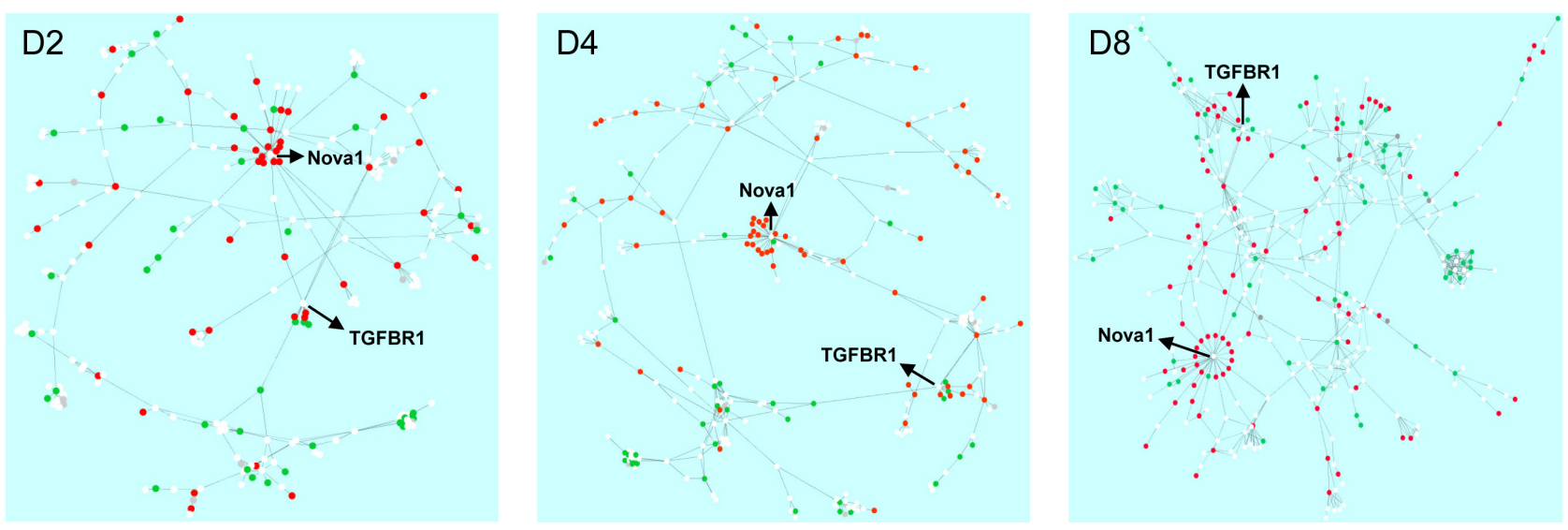

Fig. 5. Cytoscape-mediated protein interaction analysis and mapping of subnetwork involved in NPC differentiation at each time point. The network was filtered by showing nodes that were significantly upregulated (red) or downregulated (green) following 2, 4, and 8 differentiation days $(>2$ fold, $p<0.05)$ and their first neighbors (white). 
D2

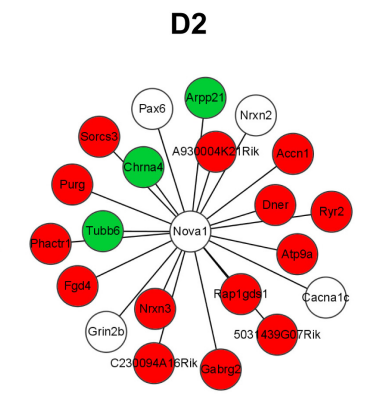

B

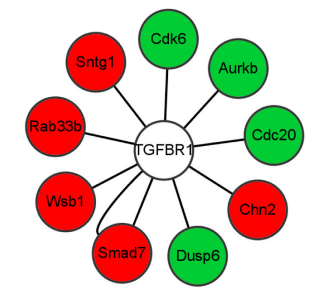

D4
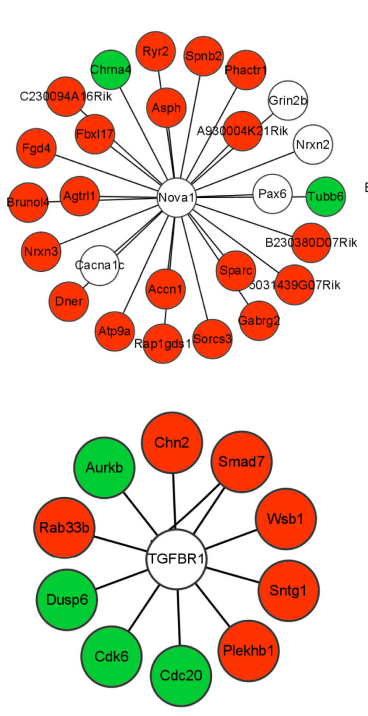
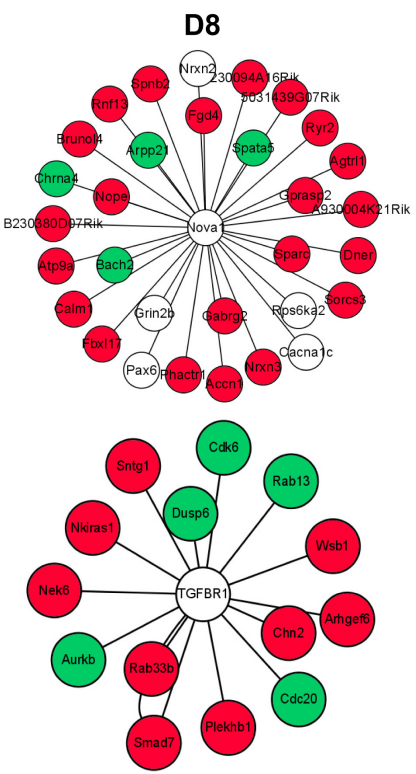

Fig. 6. Identification of nodes interacting with more than 9 genes in the subnetworks. (A) Nova1 interaction network, (B) TGFBR1 interaction network. Up- and downregulated genes are colored in red and green, respectively. in red and green, respectively. Finally, the subnetworks containing 370, 435, and 508 genes were identified for differentiation Day 2, 4, and 8, respectively (Fig. 5). As the key node (gene) among these genes, we identified 2 genes, neuro-oncological ventral antigen 1 (Nova1) and transforming growth factor beta receptor 1 (TGFBR1), which interact with more than 9 proteins in the networks.

Nova1 is reported to be necessary for the development of the motoneuron and for control of the alternative splicing of pre-mRNA involved in synapse formation and activity (Ratti et al., 2008). Because Nova1 is a neuron-specific splicing factor, the genes in the first neighbors of Nova1 were expected to be downregulated on Day 8 , the late stage of neuronal differentiation. However, most of the genes in the first neighbor of Nova1 were upregulated in a time-dependent manner along the differentiation (Fig. 6A) according to the expression analysis. Meanwhile, the expression of 6 genes (Sorcs3, Purg, Phactr1, Fgd4, Nrxn3, and Rap1gds1) in clusters 10 and 12 were found to be slightly decreased on Day 8. The network analysis showed that Nova1 has direct interactions with Pax6, BTB, and CNC homology 2 (Bach2). But Pax6 and Nova1 were filtered out in the process of DEG-finding procedures, when we performed semiquantitative reverse transcription polymerase chain reaction (RT-PCR) for each gene to check the expression level of the genes. As shown in Fig. 4, the expression of Pax6 significantly decreased on Days 4 and 8 , and the expression of Nova1 was slightly downregulated on Day 8. The network analysis indicated that Nova1 may play a pivotal role in neuronal development in the spinal cord.
The number of upregulated genes that connected with another node (TGFBR1) also increased in a time-dependent manner, through the differentiation period (Fig. 6B). This result was correlated with gene expression levels monitored by RT-PCR (Fig. 4). Recent studies have shown that TGF-beta1/SMAD signaling induces astrocyte fate commitment of neuronal precursor cells in vitro (Stipursky and Gomes, 2007; de Sampaio e Spohr et al., 2002). These observations imply that TGFBR1 has important roles in the astrogenesis of spinal cord precursor cells.

In summary, in order to identify genes involved in regulatory functions of the differentiation process, we analyzed the levels of whole gene expression during the differentiation of spinal cord NPCs. We identified 1878 genes whose expression levels were significantly changed during differentiation and categorized these genes into 12 clusters based on their patterns of expression. Computational analyses on biological function suggested that the Pax6-NeuroD6 pathways may be important in the generation of neurons and astrocytes. The interaction network analysis also sheds light on the possible pivotal roles of Nova1 and TGFbeta in neurogenesis and astrogenesis in the mouse spinal cord, respectively. In particular, further molecular studies on Nova1 will be of great help for elucidating the underlying regulation mechanism of neurogenesis/astrogenesis of NPCs.

\section{Acknowledgments}

This work was supported by a grant (06121KFDA424) from the Korea Food \& Drug Administration. 


\section{References}

Bertrand, N., Castro, D.S., and Guillemot, F. (2002). Proneural genes and the specification of neural cell types. Nat. Rev. Neurosci. 3, 517-530.

Bramblett, D.E., Copeland, N.G., Jenkins, N.A., and Tsai, M.J. (2002). BHLHB4 is a bHLH transcriptional regulator in pancreas and brain that marks the dimesencephalic boundary. Genomics 79, 402-412.

Camu, W., and Henderson, C.E. (1992). Purification of embryonic rat motoneurons by panning on a monoclonal antibody to the rat low-affinity NGF receptor. J. Neurosci. Meth. 44, 59-70.

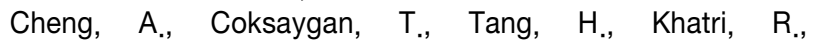
Balice-Gordon, R.J., Rao, M.S., and Mattson, M.P. (2006). Truncated tyrosine kinase B brain-derived neurotrophic factor receptor directs cortical neural stem cells to a glial cell fate by a novel signaling mechanism. $J$. Neurochem. 100, 1515-1530.

Chung, T.S., Chung, H.J., and Kim, J.H. (2007). PathTalk: Interpretation of microarray gene-expression clusters in association with biological pathways. G\&/ 5, 124-128.

Climent, E., Sancho-Tello, M., Miñana, R., Barettino, D., and Guerri, C. (2000). Astrocytes in culture express the full-length Trk-B receptor and respond to brain derived neurotrophic factor by changing intracellular calcium levels: effect of ethanol exposure in rats. Neurosci. Lett. $288,53-56$.

de Sampaio e Spohr, T.C., Martinez, R., da Silva, E.F., Neto, V.M., and Gomes, F.C. (2002). Neuro-glia interaction effects on GFAP gene: a novel role for transforming growth factor-beta1. Eur. J. Neurosci. 16, 2059-2069.

Deneen, B., Ho, R., Lukaszewicz, A., Hochstim, C.J., Gronostajski, R.M., and Anderson, D.J. (2006). The transcription factor NFIA controls the onset of gliogenesis in the developing spinal cord. Neuron 52, 953-968.

Fukuda, S., Abematsu, M., Mori, H., Yanagisawa, M., Kagawa, T., Nakashima, K., Yoshimura, A., and Taga, T. (2007). Potentiation of astrogliogenesis by STAT3-mediated activation of bone morphogenetic protein-Smad signaling in neural stem cells. Mol. Cell. Biol. 27, 4931-4937.

Guo, L., Jiang, M., Ma, Y., Cheng, H., Ni, X., Jin, Y., Xie, Y., and Mao, Y. (2002). Cloning, chromosome localization and features of a novel human gene, MATH2. J. Genet. 81, 13-17.

Holm, P.C., Mader, M.T., Aubst, H.N., Wizenmann, A., Sigvardsson, M., and Götz, M. (2007). Loss- and gain-of-function analyses reveal targets of Pax6 in the developing mouse telencephalon. Mol. Cell. Neurosci. 34, 99-119.

Jiménez, D., López-Mascaraque, L., de Carlos, J.A., and Valverde, F. (2002). Further studies on cortical tangential migration in wild type and Pax-6 mutant mice. $J$. Neurocytol. 31, 719-728.

Jones, L., López-Bendito, G., Gruss, P., Stoykova, A., and Molnár, Z. (2002). Pax6 is required for the normal development of the forebrain axonal connections. Develop- ment 129, 5041-5052.

Kageyama, R., Ohtsuka, T., Hatakeyama, J., and Ohsawa, R. (2005). Roles of bHLH genes in neural stem cell differentiation. Exp. Cell. Res, 306, 343-348

Kalyani, A., Hobson, K., and Rao, M.S. (1997). Neuroepithelial stem cells from the embryonic spinal cord: isolation, characterization, and clonal analysis. Dev. Biol. 186, 202-223.

Kalyani, A.J., Piper, D., Mujtaba, T., Lucero, M.T., and Rao, M.S. (1998). Spinal cord neuronal precursors generate multiple neuronal phenotypes in culture. J. Neurosci. 18, 7856-7868.

Kantor, D.B., Chivatakarn, O., Peer, K.L., Oster, S.F., Inatani, M., Hansen, M.J., Flanagan, J.G., Yamaguchi, Y., Sretavan, D.W., Giger, R.J., and Kolodkin, A.L. (2004). Semaphorin $5 \mathrm{~A}$ is a bifunctional axon guidance cue regulated by heparan and chondroitin sulfate proteoglycans. Neuron 44, 961-975.

Ko, M.J., Choi, H.S., Ahn, J.I., Kim, S.Y., Jeong, H.S., and Chung, H.J. (2008). Gene Expression Profiling in C57BL/6 Mice Treated with the Anorectic Drugs Sibutramine and Phendimetrazine and Their Mechanistic Implications. G\&/ 6, 117-125.

Lee, J., Wu, Y., Qi, Y., Xue, H., Liu, Y., Scheel, D., German, M., Qiu, M., Guillemot, F., Rao, M., and Gradwohl, G. (2003). Neurogenin3 participates in gliogenesis in the developing vertebrate spinal cord. Dev. Biol, 253, 84-98

Lu, Q.R., Yuk, D., Alberta, J.A., Zhu, Z., Pawlitzky, I., Chan, J., McMahon, A.P., Stiles, C.D., and Rowitch, D.H. (2000). Sonic hedgehog-regulated oligodendrocyte lineage genes encoding bHLH proteins in the mammalian central nervous system. Neuron 25, 317-329.

McLellan, A.S., Langlands, K., and Kealey, T. (2002). Exhaustive identification of human class II basic helix-loop-helix proteins by virtual library screening. Gene Expr. Patterns, 2, 329-335.

Nakashima, K., Takizawa, T., Ochiai, W., Yanagisawa, M., Hisatsune, T., Nakafuku, M., Miyazono, K., Kishimoto, T., Kageyama, R., and Taga, T. (2001). BMP2-mediated alteration in the developmental pathway of fetal mouse brain cells from neurogenesis to astrocytogenesis. Proc. Natl. Acad. Sci. U.S.A. 98, 5868-5873.

Poh, A., Karunaratne, A., Kolle, G., Huang, N., Smith, E., Starkey, J., Wen, D., Wilson, I., Yamada, T., and Hargrave, M. (2002). Patterning of the vertebrate ventral spinal cord. Int. J. Dev. Biol. 46, 597-608.

Qian, X., Shen, Q., Goderie, S.K., He, W., Capela, A., Davis, A.A., and Temple, S. (2000). Timing of CNS cell generation: a programmed sequence of neuron and glial cell production from isolated murine cortical stem cells. Neuron 28, 69-80.

Ratti, A., Fallini, C., Colombrita, C., Pascale, A., Laforenza, U., Quattrone, A., and Silani, V. (2008). Post-transcriptional regulation of neuro-oncological ventral antigen 1 by the neuronal RNA-binding proteins ELAV. J. Biol. Chem. 283, 7531-7541.

Scardigli, R., Schuurmans, C., Gradwohl, G., and Guillemot, F. (2001). Cross regulation between Neurogenin2 and 
96 Genomics \& Informatics Vol. 7(2) 85-96, June 2009

pathways specifying neuronal identity in the spinal cord. Neuron 31, 203-217.

Schwab, M.H., Bartholomae, A., Heimrich, B., Feldmeyer, D., Druffel-Augustin, S., Goebbels, S., Naya, F.J., Zhao, S., Frotscher, M., Tsai, M.J., and Nave, K.A. (2000). Neuronal basic helix-loop-helix proteins (NEX and BETA2/Neuro D) regulate terminal granule cell differentiation in the hippocampus. J. Neurosci. 20, 37143724.

Stipursky, J., and Gomes, F.C. (2007). TGF-beta1/SMAD signaling induces astrocyte fate commitment in vitro: implications for radial glia development. Glia 55, 1023-1033.

Sugimori, M., Nagao, M., Bertrand, N., Parras, C.M., Guillemot, F., and Nakafuku, M. (2007). Combinatorial actions of patterning and $\mathrm{HLH}$ transcription factors in the spatiotemporal control of neurogenesis and gliogenesis in the developing spinal cord. Development 134, 1617-1629 Sun, Y., Nadal-Vicens, M., Misono, S., Lin, M.Z., Zubiaga, A., Hua, X., Fan, G., and Greenberg, M.E. (2001). Neurogenin promotes neurogenesis and inhibits glial dif- ferentiation by independent mechanisms. Cell 104, 365376.

Sun, Y.E., Martinowich, K., and Ge, W. (2003). Making and repairing the mammalian brain signaling toward neurogenesis and gliogenesis. Semin. Cell. Dev. Biol. 14, 161-168

Xu, Z.P., Dutra, A., Stellrecht, C.M., Wu, C., Piatigorsky, J., and Saunders, G.F. (2002). Functional and structural characterization of the human gene BHLHB5, encoding a basic helix-loop-helix transcription factor. Genomics 80, 311-318.

Yue, Y., Grossmann, B., Galetzka, D., Zechner, U., and Haaf, T. (2006). Isolation and differential expression of two isoforms of the ROBO2/Robo2 axon guidance receptor gene in humans and mice. Genomics 88, 772-778.

Zhou, Q., and Anderson, D.J. (2002). The bHLH transcription factors OLIG2 and OLIG1 couple neuronal and glial subtype specification. Cell 109, 61-73. 